\title{
Antidepressant-like effects of the hydroalcoholic extracts of Hemerocallis Citrina and its potential active components
}

Bingjian Du, Xiaoshuang Tang, Fei Liu, Chunyue Zhang, Guanghua Zhao*, Fazheng Ren and Xiaojing Leng ${ }^{*}$

\begin{abstract}
Background: Herbal therapies are potential alternatives and adjuncts for depression treatment. The present study aims to investigate the antidepressant-like effects of hydroalcoholic Hemerocallis citrina extracts and its potential neuropharmacological components.

Methods: Hydroalcoholic H. citrina extracts were phytochemically analyzed. Behavioral models, including tail suspension tests and open field tests, were performed to evaluate the antidepressant-like effects of the extracts. A possible mechanism was explored by analyzing brain monoamine neurotransmitters. Toxicity and histopathological analyses were performed to determine whether or not the extracts are safe for oral administration.

Results: The antidepressant-like effects of hydroalcoholic $H$. citrina extracts were mainly related to flavonoids, especially rutin and hesperidin. The extract prepared using 75\% ethanol (i.e., HCE75) exhibited the highest active flavonoid content and activity. Orally administered $400 \mathrm{mg} / \mathrm{kg}$ of HCE75 significantly induced an antidepressant-like effect, whereas the combination of equivalent rutin and hesperidin dosages exhibited the same profiles. Isobologram analysis showed sub-additive antidepressant interactions between rutin and hesperidin. HCE75 (400 mg/kg, p.o.) increased the serotonin and dopamine levels in the central nervous system. Mortality and lesions were not observed upon oral administration of up to $5000 \mathrm{mg} / \mathrm{kg}$ HCE75.

Conclusions: The antidepressant-like effects of hydroalcoholic $\mathrm{H}$. citrina extracts are mainly related to flavonoids, especially rutin and hesperidin. The serotonergic and dopaminergic systems may have major roles. The active extract is toxicologically safe for oral administration.
\end{abstract}

Keywords: Hemerocallis citrina, Antidepressant-like effects, Neurotransmission, Flavonoids

\section{Background}

Depression is a chronic, relapsing, and potentially fatal disorder that affects about $20 \%$ of the population. Depression has been projected to become the second most common disorder worldwide by 2020 [1]. Antidepressant drugs often have undesirable side effects, such as cholinergic symptoms, withdrawal issues, sexual dysfunction [2], and worsening insomnia [3]. Therefore, developing effective depression therapies with few side effects is

\footnotetext{
* Correspondence: gzhao1000@163.com; lengxiaojingcau@163.com CAU \& ACC Joint-Laboratory of Space Food, College of Food Science \& Nutritional Engineering, Key Laboratory of Functional Dairy Science of Beijing and Ministry of Education, Beijing Higher Institution Engineering Research Center of Animal Product, Beijing Dairy Industry Innovation Team, China Agricultural University, No.17 Qinghua East Road, Haidian, Beijing 100083,
} People's Republic of China essential. Psychotropic plants that exhibit multiple bioactivity and few side effects have gained significant attention as complementary and alternative medicines $[4,5]$.

Emerging evidence suggests that plant flavonoids mainly exert beneficial effects on the central nervous system (CNS) by protecting neurons against stressinduced injury, suppressing microglia and astrocyte activation and promoting synaptic plasticity, memory, and cognitive function [6,7]. These properties depend on the chemical structure of the flavonoids. Schinus molle L. (Anacardiaceae) and Hypericum species are rich in rutin and have been reported to be antidepressants mediated by their interaction with serotonergic, noradrenergic, and dopaminergic systems [8]. The antidepressant-like 
effect of hesperidin on mice depends on its interaction with serotonergic $5-\mathrm{HT}_{1 \mathrm{~A}}$ receptors [9].

Hemerocallis citrina (daylily) is a plant widely grown in East Asia that has antibacterial [10], antioxidant [11], and nitrite-eliminating activities [12]. H. citrina has been clinically efficient in relieving depression in patients aged 11 to 80 years [13]. A previous study showed that the ethanol extract of Hemerocallis fulva has an antidepressant-like effect, in which rutin is believed to have an important role [14]. The ethanol extract of $H$. citrina has been recently reported to elicit antidepressant-like effects depending on monoaminergic systems [15]. Some researchers have also suggested that such activity of the ethanol extract is at least partly mediated by neurotrophic [16] and inflammation systems [17]. However, the relationships between specific $H$. citrina neuropharmacological activities and its flavonoid components remain uninvestigated. The scientific evaluation of its antidepressant effects are still not convincing.

In this study, the relationships between the $H$. citrina flavonoid composition and its corresponding antidepressantlike activities were determined by performing tail suspension tests, open field tests, and neurochemical analyses in mice. Toxicity and histopathological analyses were also carefully conducted.

\section{Methods}

\section{Animals}

Male imprinting control region mice (25 g to $36 \mathrm{~g}$ in weight) were purchased from Vital River Laboratories (Beijing, PR China). The mice were housed in cages and were given unrestricted access to food and water at $25 \pm$ $1^{\circ} \mathrm{C}$. Humidity was controlled at $56 \% \pm 3 \%$ in a room maintained on a $12 \mathrm{~h}$ light/dark cycle (lights on at 8 a. $\mathrm{m}$.). The mice were randomly assigned into specified experimental groups ( $\mathrm{n}=10$ animals per group) and were utilized only once. All animal procedures were conducted in accordance with the animal care and use guidelines of the China Council on Animal Care (Regulations for the Administration of Affairs Concerning Experimental Animals approved by Decree No. 2 of the State Science and Technology Commission on November 14, 1988). The experiments were approved by the Animal Experimental Welfare and Ethical Inspection Committee, The Supervision, Inspection, and Testing Center of Genetically Modified Organisms, Ministry of Agriculture (Beijing, China).

\section{Plant materials and preparation of extracts}

Dried $H$. citrina flowers $(7.3 \% \pm 1.2 \%$ moisture content; $n=6)$ were purchased from Wal-Mart (Beijing, PR China). A $100 \mathrm{~g}$ botanical sample of the flowers was finely powdered and extracted through maceration three times at
$25 \pm 2{ }^{\circ} \mathrm{C}$ for $12 \mathrm{~h}$ with $1 \mathrm{~L}$ portions of deionized water containing different ethanol concentrations (i.e., $0 \%, 25 \%$, $50 \%, 75 \%$, and $100 \%$ ). Ethanol was selected as solvent to ensure food safety. The $H$. citrina extracts (HCEs) were filtered and lyophilized at $-60^{\circ} \mathrm{C}$ for $48 \mathrm{~h}$. Freeze-dried extracts were produced using deionized water containing $0 \%$ (HCE0), 25\% (HCE25), 50\% (HCE50), 75\% (HCE75), and $100 \%$ ethanol (HCE100). The HCEs were sealed and stored in a freezer before use.

\section{Drugs and treatments}

D-glucose, bovine serum albumin, quercetin, quercitrin, isoquercitrin, rutin, hyperoside, hesperidin, serotonin (5$\mathrm{HT}$ ), norepinephrine (NE), and dopamine (DA) were provided by Sigma-Aldrich (St. Louis, MO, USA). Fluoxetine, sodium pentobarbital, and diazepam were purchased from China National Medicines (Beijing, PR China). All chemicals and reagents were analytical grade unless otherwise stated.

All experiments were performed between 13:00 and 17:00. Different groups of mice were used for each test. The HCEs were dissolved in physiological saline with $2 \%$ Tween 80; the other drugs were dissolved in physiological saline immediately before use. The reagents were orally administered (p.o.) at the constant volume of $10 \mathrm{ml} / \mathrm{kg}$ body weight. The control group mice received appropriate vehicles.

The mice subjected to the experiment for the antidepressant-like effects of HCE received behavioral tests $60 \mathrm{~min}$ after they were administered (p.o.) with the vehicle (physiological saline with $2 \%$ Tween 80), fluoxetine $(20 \mathrm{mg} / \mathrm{kg})$, and HCE $(400 \mathrm{mg} / \mathrm{kg})$. The mice were tested $60 \mathrm{~min}$ after they were administered (p.o.) with either the vehicle (physiological saline with $2 \%$ Tween 80), $20 \mathrm{mg} / \mathrm{kg}$ fluoxetine, 0.1, 1, 2, 4, and $8 \mathrm{mg} /$ $\mathrm{kg}$ rutin, $0.03,0.3,1,2$, and $4 \mathrm{mg} / \mathrm{kg}$ hesperidin, 0.01 , $0.1,0.2,0.4$, and $0.8 \mathrm{mg} / \mathrm{kg}$ quercetin, and $0.01,0.1,0.2$, 0.4 , and $0.8 \mathrm{mg} / \mathrm{kg}$ quercitrin to investigate their antidepressant effects. The mice underwent behavioral tests $0,0.5,1,2,3,4$, and $8 \mathrm{~h}$ after administering (p.o.) with the vehicle (physiological saline with $10 \%$ Tween 80 ), HCE75 (400 mg/kg), standardized flavonoid mixture $(8 \mathrm{mg} / \mathrm{kg})$, and $(75: 21.5, \mathrm{w} / \mathrm{w})$ rutin/hesperidin fixedratio combination $(8 \mathrm{mg} / \mathrm{kg})$ to investigate the temporal evolutions of the antidepressant-like effects of the treatments. The mice underwent behavioral tests $1 \mathrm{~h}$ after administration (p.o.) of the vehicle (physiological saline with $10 \%$ Tween 80 ), fluoxetine (20 mg/kg), HCE75 (20, 200, 400,800 , and $1600 \mathrm{mg} / \mathrm{kg}$ ), standardized flavonoid mixtures $(0.4,4,8,16$, and $32 \mathrm{mg} / \mathrm{kg})$, and $(75: 21.5, \mathrm{w} / \mathrm{w})$ rutin and hesperidin $(0.4,4,8,16$, and $32 \mathrm{mg} / \mathrm{kg})$ to investigate their antidepressant-like effects.

The mice of the groups that were not subjected to behavioral tests were decapitated and subjected to brain 
surgery 66 min after administration (p.o.) of the vehicle (physiological saline with $2 \%$ Tween 80), fluoxetine (20 mg/kg), and HCE75 (20, 200, 400, 800, and $1600 \mathrm{mg} /$ $\mathrm{kg}$ ) to investigate the effects of HCE75 on monoamine neurotransmitter levels in prefrontal cortex and hippocampus of mice without or with behavioral tests. The mice of the blank group were not treated, but similarly underwent brain surgery. The mice of the groups exposed to the behavioral tests were decapitated and underwent brain surgery immediately after the animals received the tests 60 min after the administration (p.o.) of the samples.

\section{Tail suspension test (TST)}

TST is a behavioral model widely used to assess antidepressant-like activities by measuring the mobility effects in each test. The immobility behavior displayed in rodents subjected to unavoidable and inescapable stresses during TST reflects behavioral despair, which reflects depression in humans [18]. The test was performed according to previously described methods [19]. In a typical procedure, acoustically and visually isolated mice were suspended $20 \mathrm{~cm}$ above the floor using adhesive tape placed approximately $1 \mathrm{~cm}$ from the tip of their tails. The total duration of immobility during a $6 \mathrm{~min}$ period was recorded. The mice were considered immobile only when they passively hung or stayed completely motionless. The video was analyzed using Smart 3.0.02 (Panlab, USA).

\section{Open-field test (OFT)}

False-positive results can be obtained in TST for agents with psychostimulant effects that could be measured through OFT [20]. The mice were evaluated in an openfield paradigm, as previously described [21]. The mice were individually placed in a Plexiglass box $(40 \mathrm{~cm} \times$ $40 \mathrm{~cm} \times 50 \mathrm{~cm}$ ) divided into 16 squares. Squares crossed with all paws were considered as indicators of locomotor activity. The behavioral parameters were recorded for $6 \mathrm{~min}$. The floor of the open-field apparatus was cleaned between tests.

\section{Phytochemical analysis of HCE}

The total carbohydrate content was measured by phenol-sulfuric acid methods using D-glucose as a standard [22]. Protein content was measured by Bradford assay, using bovine serum albumin as a standard [23]. The crude fat content was measured using the method 920.39 from the Association of Official Analytical Chemists [24]. The total flavonoid content was assessed through the aluminum chloride colorimetric method and expressed as quercetin equivalents [25]. Flavonoid profile analyses were conducted using a high-performance liquid chromatography (HPLC) system based on previous studies [26]. Qualitative and quantitative analyses were
Table 1 Chemical composition of HCE $(\%, w / w)$

\begin{tabular}{llllll}
\hline Sample & Yield & Carbohydrate & Protein & Crude fat & Flavonoid \\
\hline HCE0 & $18.2 \pm 1.2$ & $80.1 \pm 4.1$ & $9.2 \pm 0.6$ & $0.3 \pm 0.1$ & $0.4 \pm 0.1$ \\
HCE25 & $16.9 \pm 0.9$ & $81.8 \pm 3.3$ & $8.5 \pm 0.4$ & $1.1 \pm 0.2$ & $0.6 \pm 0.1$ \\
HCE50 & $14.9 \pm 0.8$ & $75.2 \pm 2.9$ & $8.1 \pm 1.0$ & $1.7 \pm 0.3$ & $0.7 \pm 0.1$ \\
HCE75 & $9.2 \pm 0.6$ & $69.4 \pm 5.3$ & $6.7 \pm 0.7$ & $6.3 \pm 0.7$ & $2.0 \pm 0.2$ \\
HCE100 & $4.4 \pm 0.5$ & $11.4 \pm 0.8$ & $0.2 \pm 0.1$ & $74.1 \pm 2.1$ & $2.3 \pm 0.2$ \\
\hline
\end{tabular}

Data are expressed as mean \pm S.E.M. $(n=3)$.

performed using a Purospher STAR RP-18 column $(150 \mathrm{~mm} \times 4.6 \mathrm{~mm}, 5 \mu \mathrm{m})$. Solvents A (acetic acid-water, 5:95) and $B$ (pure methanol) were programmed at $0(95 \%$ A + 5\% B), 17 (88.5\% A + 11.5\% B), 28 (88.3\% A + 11.7\% B), $57(28.3 \% \mathrm{~A}+71.7 \% \mathrm{~B})$, and $60 \mathrm{~min}(95 \% \mathrm{~A}+5 \% \mathrm{~B})$, at $0.8 \mathrm{ml} / \mathrm{min}$ flow rate for chromatography. The column temperature was maintained at $40^{\circ} \mathrm{C}$, the injection volume was $10 \mu \mathrm{l}$, and the detection wavelength was $254 \mathrm{~nm}$. The flavonoid compounds were identified by comparing the retention time with authentic standards, and quantitative analysis was performed in triplicate to obtain external calibration curves.

\section{Isobologram analysis}

Synergy was graphically assessed through isobologram analysis [27]. In brief, the median effective doses of the drugs and their combinations were determined at a fixed ratio; dose-response curves were plotted in rectangular coordinates. The straight line connecting the points of the drugs represents their theoretical additive combination. The effects of the drugs are purely additive (no interaction) when the point of their combination and their S.E.M. lie on this line. Super-additivity exists when the points lie below this line, but sub-additivity (antagonism) exists when the points lie above this line.

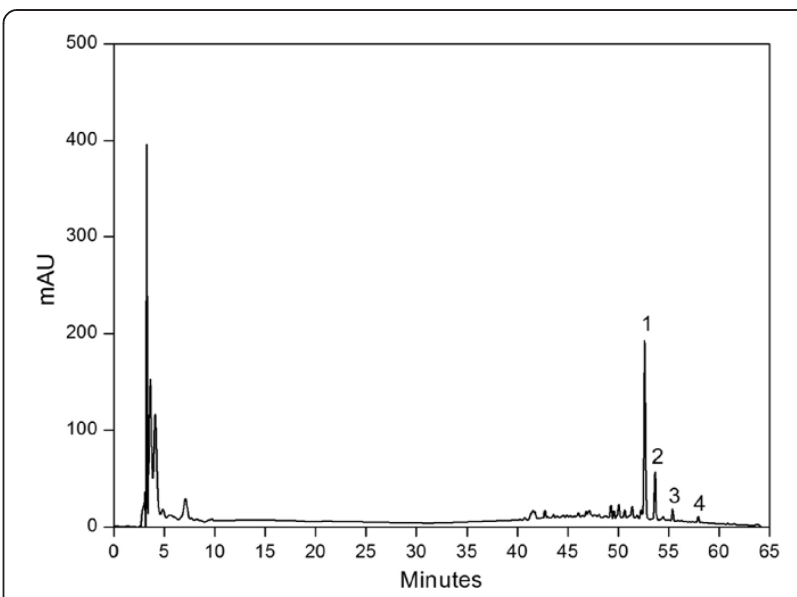

Figure 1 Flavonoid fingerprint of HCE75 as a representative HPLC chromatogram. The peaks correspond to (1) rutin, (2) hesperidin, (3) quercitrin, and (4) quercetin. 
Table 2 Composition of the HCE flavonoids (\%, w/w)

\begin{tabular}{|c|c|c|c|c|c|c|}
\hline Sample & Quercetin & Quercitrin & Isoquercitrin & Rutin & Hyperoside & Hesperidin \\
\hline HCEO & $0.8 \pm 0.1$ & $0.6 \pm 0.1$ & $\mathrm{ND}$ & $96.2 \pm 5.7$ & ND & $0.2 \pm 0.0$ \\
\hline HCE25 & $5.9 \pm 1.0$ & $3.8 \pm 0.2$ & ND & $73.2 \pm 1.9$ & ND & $15.2 \pm 2.1$ \\
\hline HCE50 & $3.7 \pm 0.9$ & $1.3 \pm 0.4$ & ND & $65.7 \pm 8.4$ & ND & $28.1 \pm 5.3$ \\
\hline HCE75 & $1.5 \pm 0.4$ & $2.1 \pm 0.3$ & ND & $74.2 \pm 6.8$ & ND & $21.3 \pm 3.7$ \\
\hline HCE100 & $3.3 \pm 0.7$ & $3.7 \pm 0.5$ & ND & $69.2 \pm 5.9$ & ND & $24.1 \pm 2.4$ \\
\hline
\end{tabular}

Data are expressed as mean \pm S.E.M. $(n=3)$; ND, not determined.

\section{Monoamine neurotransmitter levels}

The 5-HT, NA, and DA concentrations in the mouse brain were measured through HPLC with electrochemical detection, as previously described [28]. The mice were decapitated, and their brains were immediately removed. The frontal cortex and hippocampus were carefully dissected and stored at $-80^{\circ} \mathrm{C}$ until measurement (no more than 1 week). The brain tissues were homogenized in an ice-cold $0.4 \mathrm{M}$ perchloric acid $(5 \mu \mathrm{l} / \mathrm{mg})$ solution that contains $5 \mathrm{mM}$ sodium bisulfate and $0.04 \mathrm{mM}$ EDTA to avoid oxidation. The homogenate was centrifuged at $15,000 \times g$ for $15 \mathrm{~min}$ at $4^{\circ} \mathrm{C}$. Approximately $10 \mu \mathrm{l}$ of the resulting supernatant was chromatographed on a Purospher STAR RP-18 column $(150 \mathrm{~mm} \times 4.6 \mathrm{~mm}$,

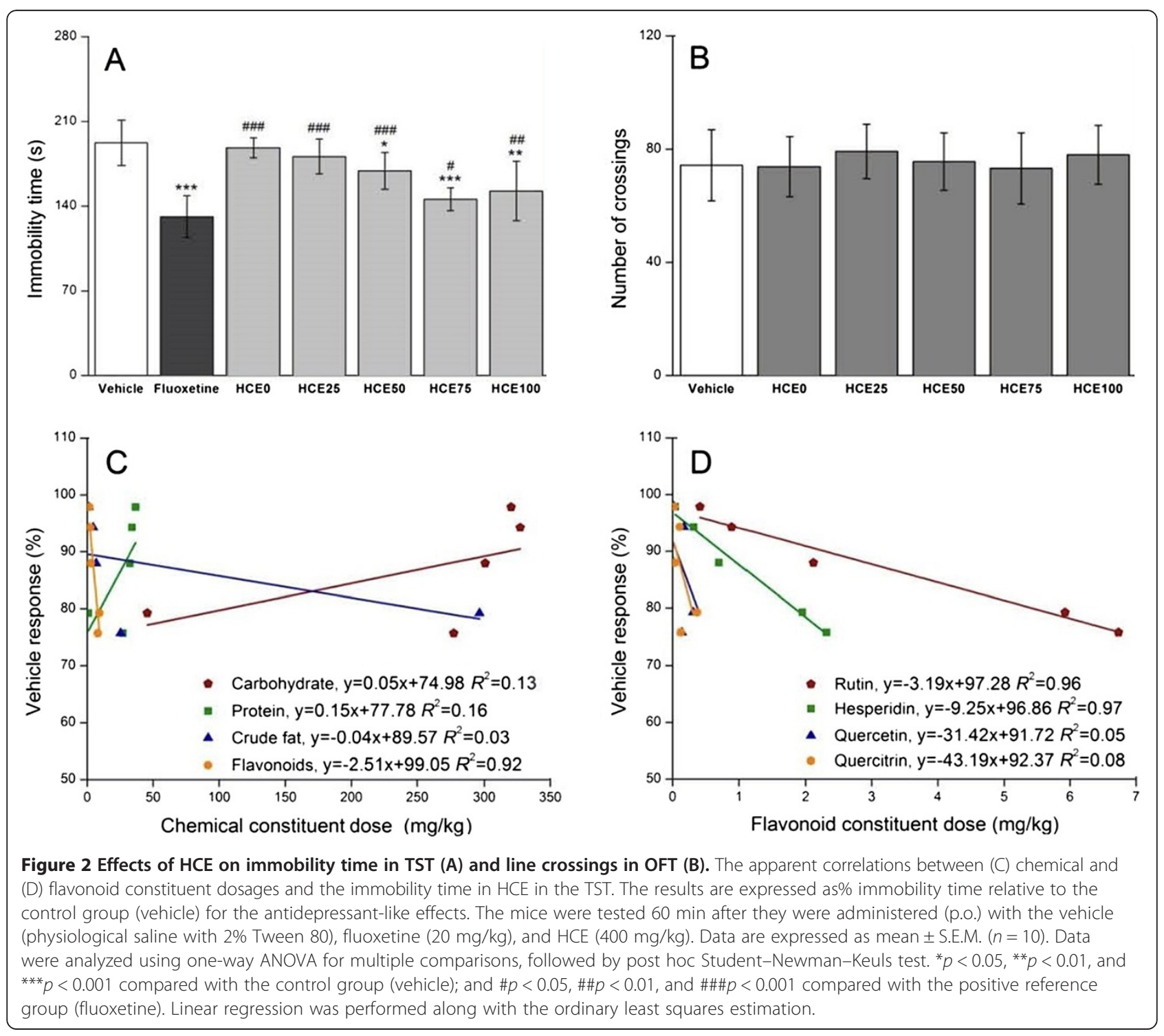


$5 \mu \mathrm{m})$. Separation was performed in an isocratic elution mode using a mobile phase at $30^{\circ} \mathrm{C}$ column temperature. The phase comprised $8 \%$ methanol and $92 \%$ water, containing EDTA (0.5 mM), triethylamine ( $5 \mathrm{mM})$, sodium 1heptanesulfonate $(0.5 \mathrm{mM}), \mathrm{Na}_{2} \mathrm{HPO}_{4}{ }^{\circ} 12 \mathrm{H}_{2} \mathrm{O}(20 \mathrm{mM})$, and citrate $(50 \mathrm{mM})$, at $1 \mathrm{ml} / \mathrm{min}$ flow rate. The neurotransmitters were measured using the electrode potential of a glassy carbon working electrode at $+650 \mathrm{mV}$ against the $\mathrm{Ag} / \mathrm{AgCl}$ electrode. The 5-HT, NA, and DA were identified and quantified by comparing their retention times and peak areas with those of standard solutions; their contents were expressed as ng/g wet weight tissue.

\section{Acute toxicity and histopathological analysis}

Orally acute HCE toxicity was estimated according to the procedure reported by Lorke [29]. Each of the three different animal groups ( $n=3$ animals per group) were administered (p.o.) with 10, 100, and $1000 \mathrm{mg} / \mathrm{kg}$ of extract during the first phase. The doses were then increased to 1600,2900 , and $5000 \mathrm{mg} / \mathrm{kg}$ in another three different groups ( $\mathrm{n}=3$ animals per group) when mortality was not observed during the first day of the first phase and 1 week thereafter. The mice were observed for $14 \mathrm{~d}$ to note possible mortality.

Histopathological analysis was conducted in a separate series of experiments. Mice ( $\mathrm{n}=10$ animals per group) were orally treated with $5000 \mathrm{mg} / \mathrm{kg}$ HCE75 once a day for $1 \mathrm{~d}$ or 21 consecutive days, and the mice were sacrificed for histopathological analyses according to the previously described methods [30]. Livers, kidneys, and spleens were immediately collected and placed in formalin. The samples were transferred to a cassette and immersed in multiple baths containing progressively higher volumes of ethanol to dehydrate the tissue, followed by xylene and

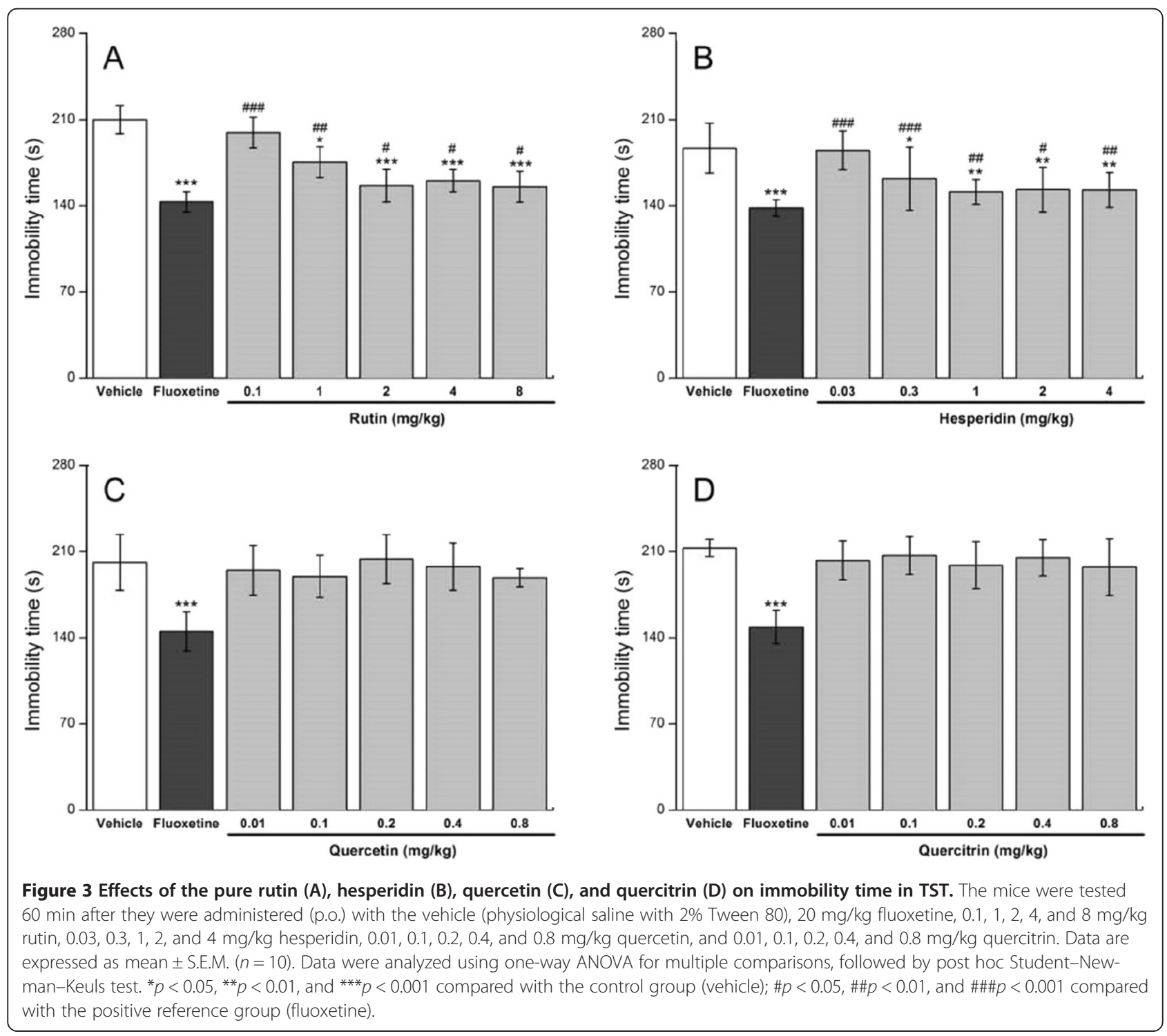


hot paraffin. More paraffin was added during embedding to create a paraffin block, which allowed the sectioning of the tissues into $2 \mu \mathrm{m}$ slices. The microtome slices were stained with hematoxylin-eosin stain.

\section{Statistical analysis}

All values were expressed as mean \pm S.E.M. The data were analyzed using ANOVA, followed by post hoc Student-Newman-Keuls test for multiple comparisons. Statistically significant differences were denoted by $p<0.05$. Linear regression was performed along with the ordinary least squares estimation.

\section{Results and discussion}

\section{Phytochemical profile analysis of HCE}

The HCE0, HCE25, HCE50, HCE75, and HCE100 extraction yields and their main compositions are shown in Table 1. Increasing ethanol concentration in the extracting solvents decreased the carbohydrate and protein contents and increased the crude fat and flavonoid contents in the extract. The increase in crude fat content in HCE100 indicates the enhanced fat-soluble compound extraction by pure ethanol. Nevertheless, the extracted fat-soluble compounds prevent the further extraction of the flavonoids [31]. The extracted flavonoid in HCE100 did not rapidly increase.

The flavonoid components of HCE were analyzed through HPLC. Figure 1 illustrates the representative chromatogram of HCE. Peaks 1, 2, 3, and 4 correspond to rutin $(52.6 \mathrm{~min})$, hesperidin $(53.2 \mathrm{~min})$, quercitrin (54.8 $\mathrm{min}$ ), and quercetin (57.9 $\mathrm{min})$, respectively. Rutin is the major component of $\mathrm{HCE}$, followed by hesperidin. The quercitrin and quercetin contents were relatively minor. Table 2 lists the main flavonoid components.

\section{Antidepressant-like effects of HCE and neuropharmacological component analysis}

The effects of HCE treatment (400 mg/kg, p.o.) on immobility time in TST are presented in Figure 2A. HCE50 $(p<0.05)$, HCE75 $(p<0.001)$, and HCE100 $(p<$ $0.01)$ significantly decrease immobility time compared with the control group, indicating good antidepressantlike performances. The reducing abilities of HCE75 were closest among the three to that of fluoxetine, which is the most common clinical therapy drug used as positive reference. Significant variations in OFT line crossings were not found in Figure 2B, indicating that HCE did not stimulate locomotor activity, thus the antidepressant-like effects of HCE in the TST were specific. The poor anti-immobility correlations with the carbohydrates $\left(R^{2}=0.16\right)$, proteins $\left(R^{2}=0.13\right)$, and crude fat $\left(R^{2}=0.03\right)$, unlike flavonoids $\left(R^{2}=0.92\right)$, derived from their dose-response regression analyses shown in Figure $2 \mathrm{C}$ indicate a possible disconnect with any antidepressant-like effect [32]. Quercetin $\left(R^{2}=\right.$ $0.05)$ and quercitrin $\left(R^{2}=0.08\right)$ were also ruled out in the regression analyses because of the apparent noncorrelation between the flavonoids and antidepressant-like performance (Figure 2D). By contrast, the high degree of correlation between immobility time and dose indicates that the antidepressant-like effects are closely associated with rutin $\left(R^{2}=0.96\right)$ and hesperidin $\left(R^{2}=\right.$ 0.97).

Pure flavonoids were tested to confirm the hypothesis. Figure $3 \mathrm{~A}$ and $3 \mathrm{~B}$ show that pure rutin $(2-8 \mathrm{mg} / \mathrm{kg})$ and hesperidin $(1-4 \mathrm{mg} / \mathrm{kg}$ ) elicits significant antidepressantlike actions on mice in TST, similar to that evoked by the equivalent HCE50 (2.1 and $0.7 \mathrm{mg} / \mathrm{kg}$ rutin and hesperidin, respectively), HCE75 (6.7 and $2.3 \mathrm{mg} / \mathrm{kg}$ rutin and hesperidin, respectively), and HCE100 (5.9 and $1.9 \mathrm{mg} / \mathrm{kg}$ rutin and hesperidin, respectively). The orally administered quercetin and quercitrin doses did not exhibit antidepressant-like effects in the present conditions (Figure 3C and 3D). Traces of contributions from carbohydrates, proteins, and crude fat were not observed. Psychostimulant effects were likewise not observed in the OFT of the flavonoids (data not shown). Dimpfel [33] found that 5 to $80 \mathrm{mg} / \mathrm{kg}$ rutin doses produced electropharmacograms similar to those of clinical antidepressants in rats. A single i.p. hesperidin administration yielded antidepressant-like effects [9].

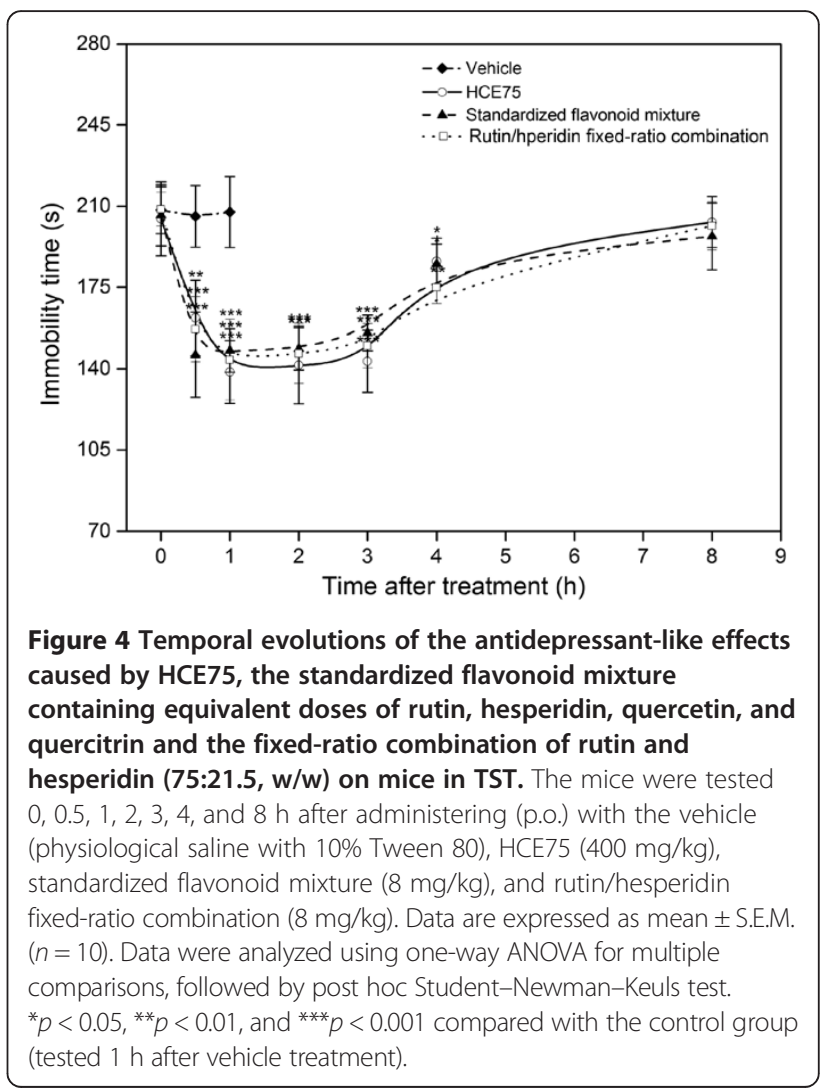




\section{Antidepressant-like effect of HCE75 and active flavonoid involvement}

The previous analysis reveals that HCE75 is the most active $H$. citrina extract. The temporal behavior of the antidepressant-like effects and the dose-dependent properties $(100 \mathrm{mg} / \mathrm{kg}$ to $1600 \mathrm{mg} / \mathrm{kg}$ ) of the extract was estimated. The standardized flavonoid mixture prepared using four types of pure flavonoids and the combination with pure rutin and hesperidin at a fixed ratio (75:21.5, w/ w) with respect to the real flavonoid composition of HCE75 (75\% rutin, 21.5\% hesperidin, 2\% quercetin, and $1.5 \%$ quercitrin, w/w) were compared. Figure 4 shows the comparison of the three samples. The antidepressant-like effects of HCE75, standardized flavonoid mixture, and rutin/hesperidin fixed-ratio combination reached their maxima $(p<0.001)$ at $1,0.5$, and $0.5 \mathrm{~h}$, respectively. The anti-depressant effects remained significant $(p<0.01)$ up to $4 \mathrm{~h}$ after p.o. administration. The temporal evolution curves were consistent. Psychostimulant effects were not observed in OFT (data not shown). Therefore, the functionality of the major antidepressants in HCE75 is mediated by rutin and hesperidin, whereas the contributions of quercetin and quercitrin are insignificant.

HCE75 (200-800 mg/kg) exhibited a significant antiimmobility effect $(p<0.01)$ in TST compared with the control groups (Figure 5A). Doses more than $800 \mathrm{mg} / \mathrm{kg}$ increased immobility time and formed a U-shaped dose-response profile in antidepressant-like action. Insignificant variations in the OFT line crossings (Figure 5B) ruled out the psychostimulant and sedative effects. The phenomenon was often observed during rodent model screening (i.p. or p.o.) on potential antidepressants, which
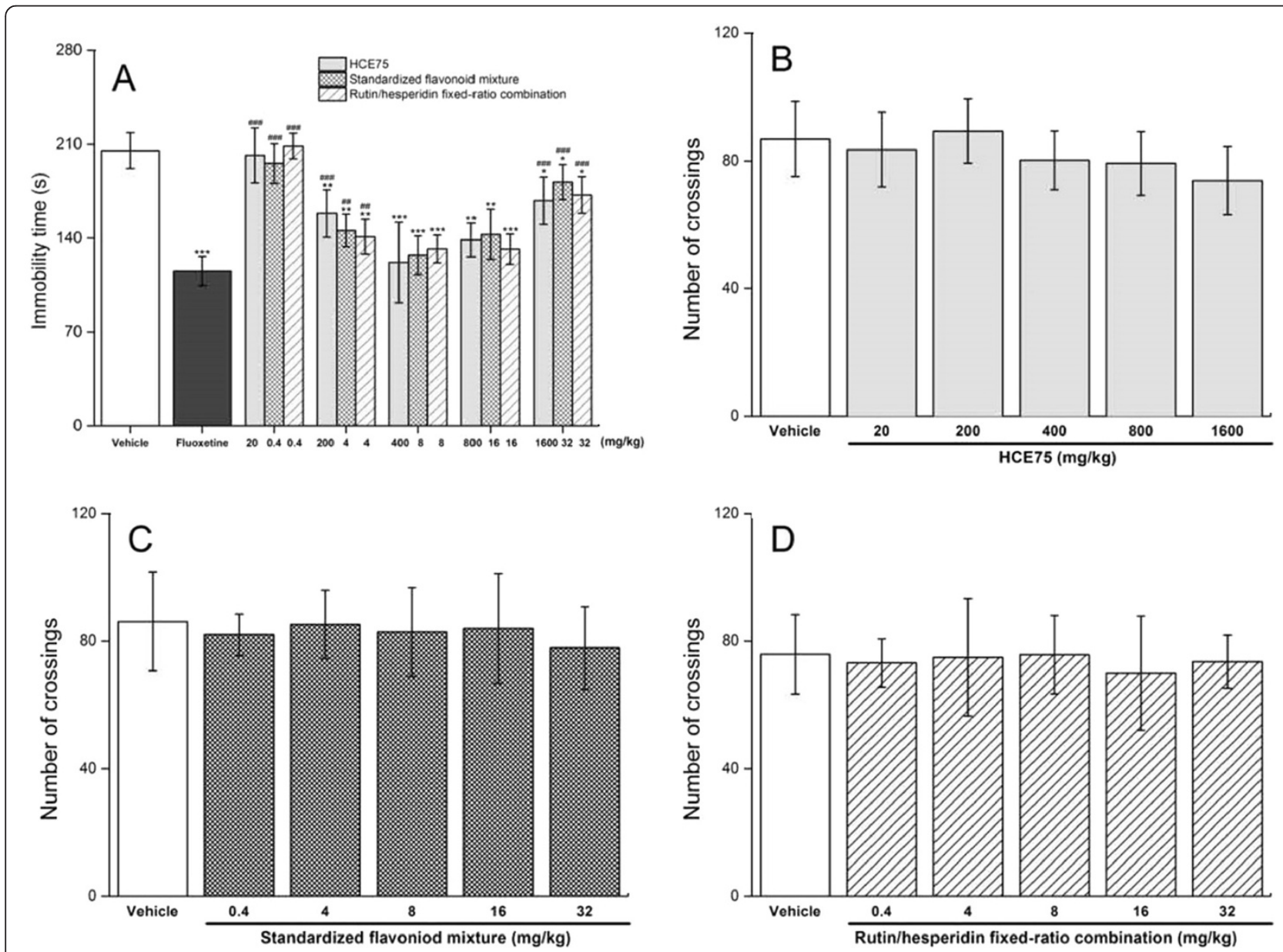

Figure 5 Effects of HCE75, the standardized flavonoid mixture containing equivalent rutin, hesperidin, quercetin, and quercitrin doses and the fixed-ratio combination of rutin and hesperidin $(75: 21.5, \mathrm{w} / \mathrm{w})$, on (A) immobility time in TST and (B to D) their respective line crossings in OFT. The mice were tested $1 \mathrm{~h}$ after administration (p.o.) of the vehicle (physiological saline with 10\% Tween 80), fluoxetine

(20 mg/kg), HCE75 (20, 200, 400, 800, and $1600 \mathrm{mg} / \mathrm{kg})$, standardized flavonoid mixtures $(0.4,4,8,16$, and $32 \mathrm{mg} / \mathrm{kg}$ ), and the fixed-ratio rutin and hesperidin combination $(0.4,4,8,16$, and $32 \mathrm{mg} / \mathrm{kg})$. Data are expressed as mean \pm S.E.M. $(n=10)$. Data were analyzed using one-way ANOVA for multiple comparisons, followed by post hoc Student-Newman-Keuls test. ${ }^{*} p<0.05,{ }^{* *} p<0.01$, and ${ }^{* * *} p<0.001$ compared with the control group (vehicle); \#\#p $<0.01$ and \#\#\#p 0.001 compared with the positive reference group (fluoxetine). 
was attributed to either the activation of different pathways at diverse doses [34] or the suppression of the maximum response in the presence of antagonists through non-competitive antagonism [4]. The dose-dependent diversity of the mechanisms of the neuropharmacological effects should be studied further because the highest HCE75 dosage (i.e., $1600 \mathrm{mg} / \mathrm{kg}$ ) for oral treatment resulted in a hypnotic response (data not shown). The response might negate the antidepressant-like effects.

The standardized flavonoid mixture containing equivalent rutin, hesperidin, quercetin, and quercitrin doses and the fixed-ratio combination of rutin and hesperidin induced the same characteristics and changes without significantly changing the OFT line crossings (Figure 5C and 5D). These results indicate that rutin and hesperidin cause the antidepressant effects of HCE75. The contributions of quercetin and quercitrin in the effects were insignificant.

Dose-response curves were obtained for rutin, hesperidin, and their fixed-ratio combination (75:21.5, w/w; Figure $6 \mathrm{~A}$ ) to assess the interaction between rutin and hesperidin in the antidepressant-like effects in TST. The data were fitted to logistic dose-response curves via nonlinear regression. Dose-dependent antidepressant-like effects were observed for the chosen dose ranges with $2.5 \mathrm{mg} / \mathrm{kg}$ rutin, $1.2 \mathrm{mg} / \mathrm{kg}$ hesperidin, and $3.7 \mathrm{mg} / \mathrm{kg}$ fixed-ratio rutin/hesperidin combination median effective dose values. The isobolograms obtained for the rutin/hesperidin fixed-ratio combination (Figure 6B) reveal a sub-additive effect. The result implies an antagonistic interaction between rutin and hesperidin. Few studies focused on the assessment of the neuroactive properties of flavonoid-flavonoid interactions. The active potency of a given flavonoid is closely linked to its structure, such as the ortho-dihydroxy structure in the B-ring, the 2-3-double bond in conjugation with a 4-oxo function, and the presence of the 3- and 5-OH functions [35]. More detailed studies are required to elucidate the mechanisms involved in the neuropharmacological interactions.

\section{Effects of HCE75 on monoamine neurotransmitter levels in mice brains}

Depression symptoms are associated with changes in monoamine neurotransmitter (i.e., NE, DA, and 5-HT) levels in the CNS $[1,36]$. The prefrontal cortex and hippocampus, which regulate emotion, motivation, learning, and memory, are essential in depression [37]. Nutt [36] revealed that NE is related to alertness, energy, and attention, and that DA is linked to pleasure, reward, and motivation in life. The 5-HT transmitter is related to compulsion, obsession, and anxiety.

Figure 7 shows the monoamine neurotransmitter levels (i.e., NE, DA, and 5-HT) in the prefrontal cortex (Figure 7A and 7C) and hippocampus (Figure 7B and $7 \mathrm{D}$ ) of the mice without (Figure $7 \mathrm{~A}$ and $7 \mathrm{~B}$ ) or with TST (Figure 7C and 7D), respectively, after treatment. The DA level in the hippocampus was too low to be detected.

Insignificant differences were observed in the monoamine neurotransmitter levels in the prefrontal cortex (Figure 7A) or hippocampus (Figure 7B) of the blank, vehicle, fluoxetine, and HCE75 treatment groups without TST. The NE levels of HCE75-treated groups with TST were almost similar to that of the vehicle, and the levels significantly $(p<$ $0.05)$ decreased after exposure to the behavioral models. Anti-reduction was observed in the fluoxetine-treated group (Figure 7D). DA and 5-HT levels were significantly higher $(p<0.05)$ with TST and the HCE75 dose of more
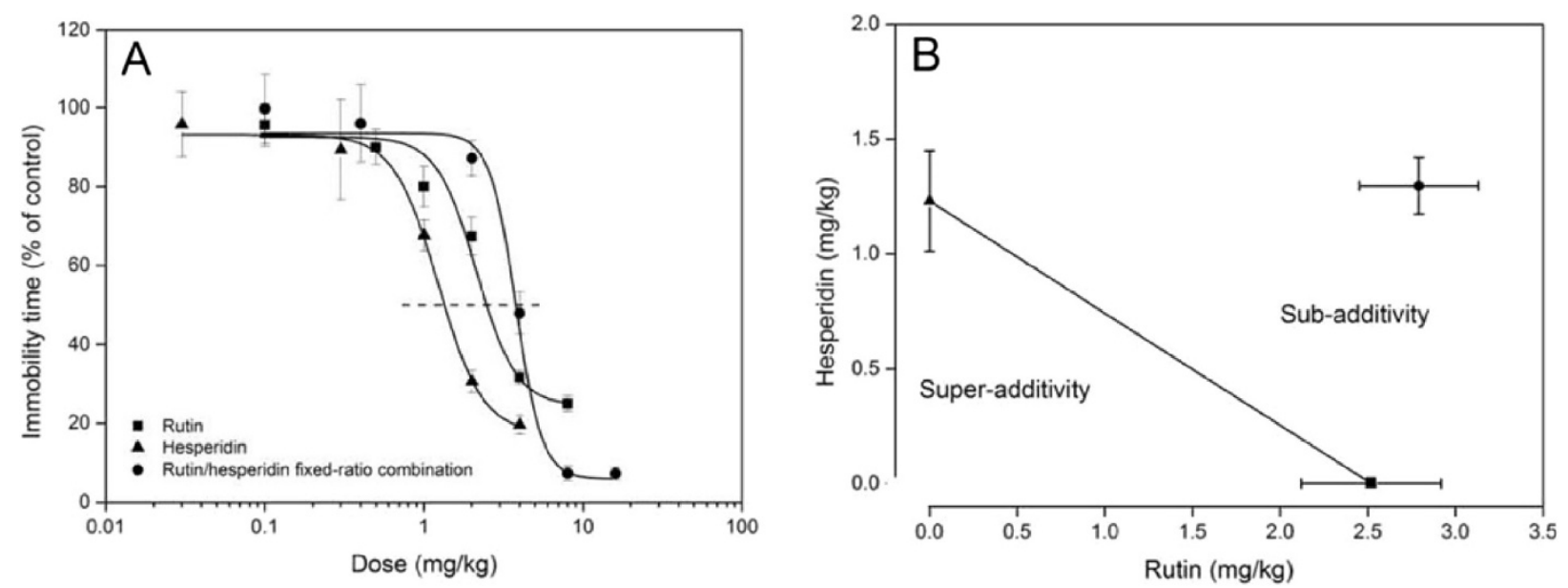

Figure 6 Dose-response curves of the immobility time in TST with respect to the control groups. (A) The positive reference group (fluoxetine, $20 \mathrm{mg} / \mathrm{kg}$, p.o.) response was 0 for the antidepressant-like effects of rutin, hesperidin, and the fixed-ratio combination of rutin/hesperidin (75:21.5, w/w). (B) The consequent isobolograms. Data are expressed as mean \pm S.E.M. $(n=10)$. 


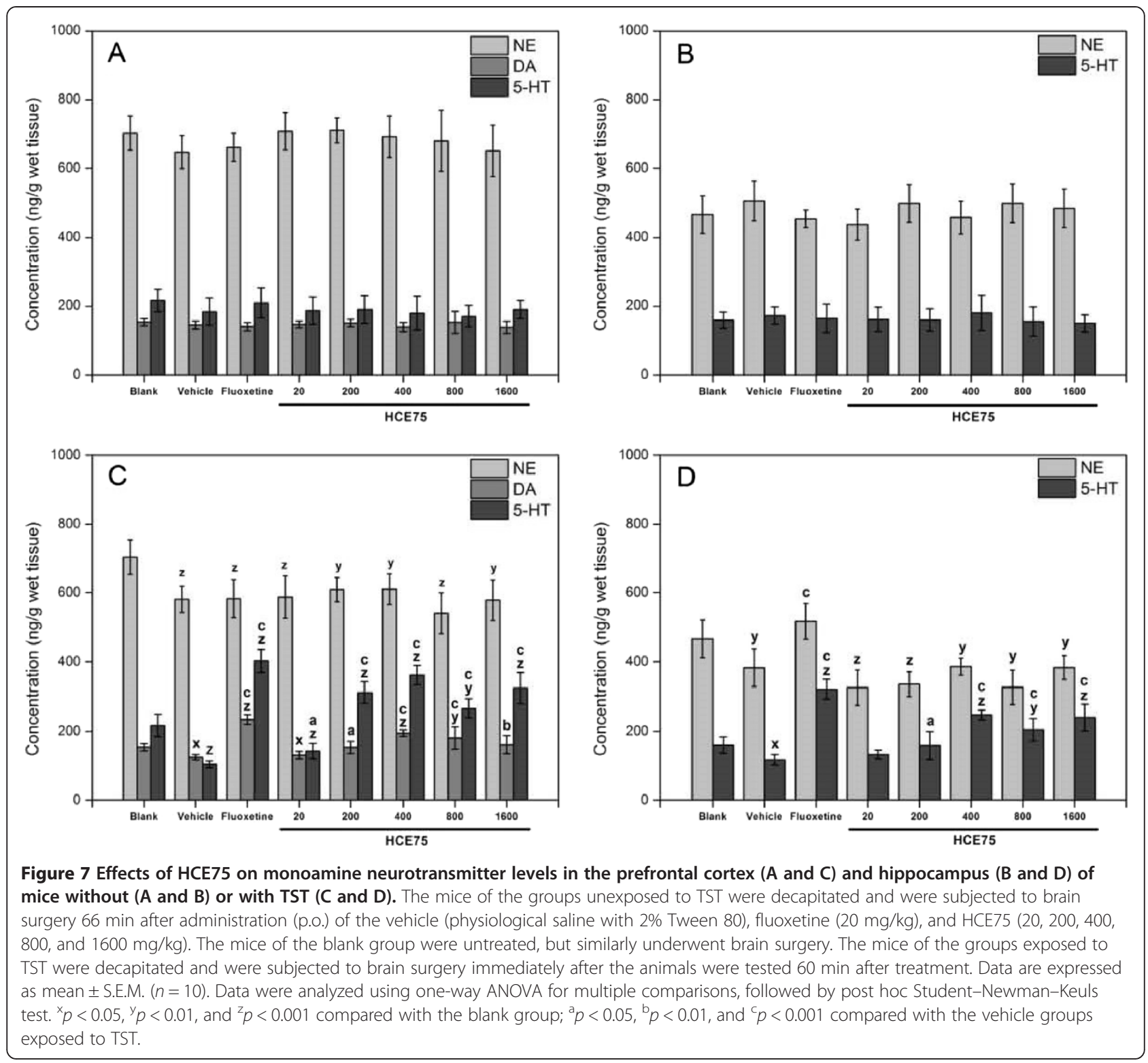

than $200 \mathrm{mg} / \mathrm{kg}$ than the vehicle groups. These levels reached their maxima at $400 \mathrm{mg} / \mathrm{kg}$ in almost all cases, and were maintained for doses up to $1600 \mathrm{mg} / \mathrm{kg}$, the highest dose tested in the present work. This point is consistent with the anti-immobility effects of HCE75 described in the previous section. This condition indicates that increasing the 5-HT and DA levels in the CNS is an important parameter in the mechanism of the antidepressant-like response of HCE75. Machado et al. [8] proved the involvement of 5-HT, DA, and/or NE systems in the antidepressant-like action of rutin. The antidepressant-like performance of hesperidin was determined to be related to the 5 -HT system, whereas NE and DA systems were indirectly involved [9], consistent with the findings in this study. However, the potential interaction between rutin and hesperidin requires further study. The U-shaped dose-response profile observed in the previous section should not be due to the decrease in antidepressant effect of HCE75, because the increasing effects of HCE75 at doses ranging from $400 \mathrm{mg} / \mathrm{kg}$ to $1600 \mathrm{mg} / \mathrm{kg}$ on the monoamine neurotransmitter levels in the TST were basically stable.

\section{Toxicity and histopathological analysis}

Plants rich in neuroactive flavonoids are usually inedible, which makes the consideration of safety and tolerability a requirement in plant supplement development. The toxicity study on HCE75 by Lorke [29] revealed that the median lethal dose is not feasibly estimated because mortality was not recorded from the oral administration of $5000 \mathrm{mg} / \mathrm{kg}$ HCE75. 

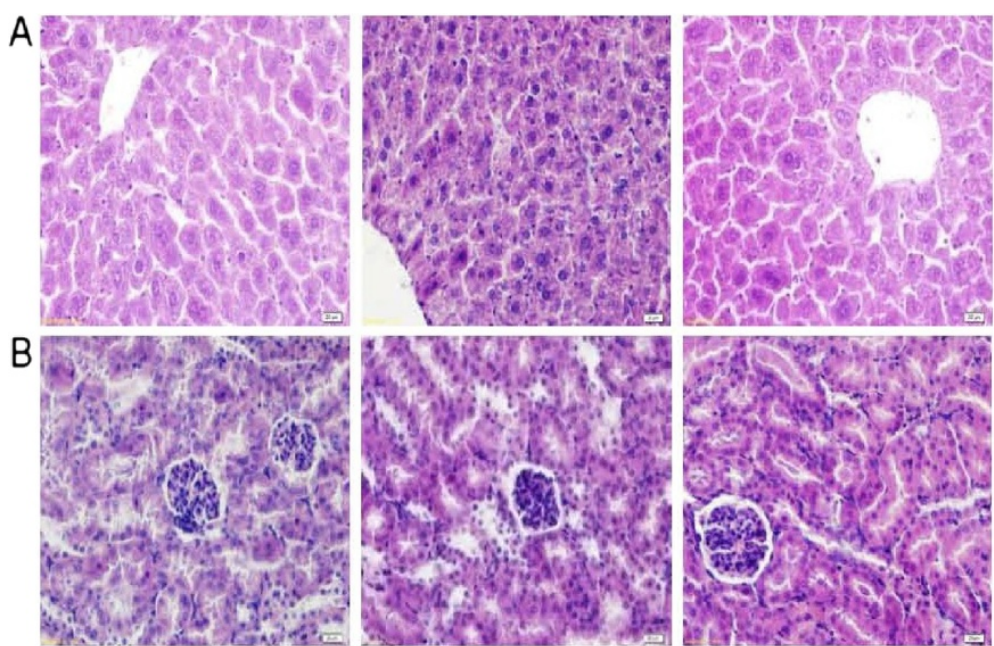

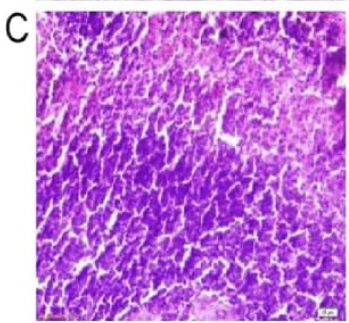

Control

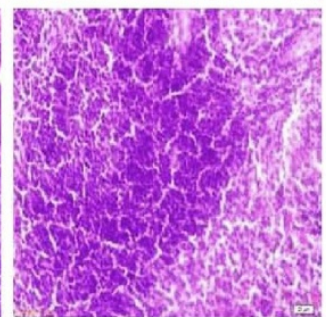

Acute treatment

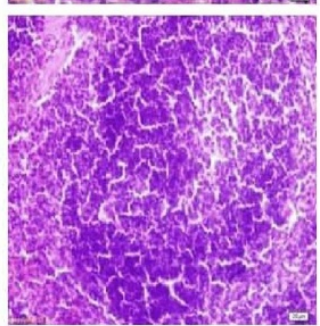

Chronic treatment

Figure 8 Representative histopathological sections of the (A) liver, (B) kidney, and (C) spleen of the mice treated with HCE75 ( $5000 \mathrm{mg} / \mathbf{k g}$, p.o.) once a day for $1 \mathrm{~d}$ (acute treatment) or 21 consecutive days (chronic treatment). The control group was not treated. Insignificant lesions were observed in the experiments. Hematoxylin-eosin stains, $400 \times$ original magnification.

Figure 8 shows that the histopathological examination has insignificant differences. The results reveal that HCE75 (5000 $\mathrm{mg} / \mathrm{kg}$, p.o.) administration once a day either for $1 \mathrm{~d}$ or 21 consecutive days does not cause lesions on the examined physiological organs. Oral HCE75 administration per se could thus be considered toxicologically safe.

\section{Conclusions}

In summary, the present TST, OFT, and neurochemical analyses of the monoamine neurotransmitters in mice brain revealed the significant antidepressant effects of $H$. citrina. The effect of HCE75, the most active hydroalcoholic extract of $H$. citrina, is dose-dependent. The results confirm that such antidepressant effect is mainly related to the contributions of flavonoids, especially rutin and hesperidin. Isobologram analysis showed the sub-additive interaction between rutin and hesperidin. Toxicity and histopathological analyses confirmed that HCE75 is toxicologically safe for oral administration.

\section{Competing interests}

The authors declare that they have no competing interests.

\section{Authors' contributions}

$\mathrm{BD}$ designed the study, performed experiments, analyzed results and contributed to writing of the paper. XT, FL and CZ helped to perform experiments. GZ, FR and XL contributed to scientific discussion and edited manuscript. All authors read, edited and ultimately approved the final manuscript.

\section{Acknowledgments}

This research was supported by the National Natural Science Foundation of China (No. 31171771), the National Science and Technology Support

Program (2011BAD23B04), and Beijing Dairy Industry Innovation Team.

Received: 25 April 2014 Accepted: 29 August 2014

Published: 1 September 2014

\section{References}

1. Krishnan V, Nestler EJ: The molecular neurobiology of depression. Nature 2008, 455(7215):894-902

2. Papakostas G: Tolerability of modern antidepressants. J Clin Psychiatr 2007, 69:8-13.

3. Pack Al, Pien GW: Update on sleep and its disorders. Annu Rev Med 2011, 62:447-460

4. Huerta-Reyes M, Herrera-Ruiz M, González-Cortazar M, Zamilpa A, León E, Reyes-Chilpa R, Aguilar-Rojas A, Tortoriello J: Neuropharmacological in vivo effects and phytochemical profile of the extract from the aerial parts of Heteropterys brachiata (L.) DC. (Malpighiaceae). J Ethnopharmacol 2013, 146(1):311-317.

5. McClatchey WC, Mahady GB, Bennett BC, Shiels L, Savo V: Ethnobotany as a pharmacological research tool and recent developments in CNS-active natural products from ethnobotanical sources. Pharmacol Ther 2009, 123(2):239-254.

6. Spencer J: Flavonoids: modulators of brain function. Br J Nutr 2008, 99(Suppl 1):ES60-ES77.

7. Jäger AK, Saaby L: Flavonoids and the CNS. Molecules 2011 16(2):1471-1485

8. Machado DG, Bettio LE, Cunha MP, Santos AR, Pizzolatti MG, Brighente I, Rodrigues ALS: Antidepressant-like effect of rutin isolated from the 
ethanolic extract from Schinus molle L. in mice: Evidence for the involvement of the serotonergic and noradrenergic systems. Eur J Pharmacol 2008, 587(1):163-168.

9. Souza LC, de Gomes MG, Goes AT, Del Fabbro L, Boeira SP, Jesse CR Evidence for the involvement of the serotonergic $5-\mathrm{HT}_{1 \mathrm{~A}}$ receptors in the antidepressant-like effect caused by hesperidin in mice. Prog NeuroPsychopharmacol Biol Psychiatry 2013, 40:103-109.

10. Zhan L, Li G, Li S, Wu S: Study on Extraction of Flavonids in Hemerocallis Citrina Baroni and Its Medical Effects. J Nanhua Univ (Medical Edition) 2005, 1:036.

11. Lang N, Luo H: Study for the activity of flavonoid in Hemerocallis citrina. Food Res Dev 2007, 28:74-77.

12. Fu M, Chen Q, Mao L: Suppression of nitrosation by extracts of nightlily (Hemerocallis citrina) flowers. Food Sci 2009, 30:114-119.

13. Chen G, Zhang C, Fan Y, Chen J: Treatment of 156 cases of depression related insomnia by Hemerocallis citrina. Zhejiang J Traditional Chinese Med 2008, 43:397.

14. Lin S, Chang H, Chen P, Hsieh C, Su K, Sheen L: The Antidepressant-like Effect of Ethanol Extract of Daylily Flowers (Jīn Zhēn Huā) in Rats. J Tradit Complement Med 2013, 3(1):53.

15. Gu L, Liu Y, Wang Y, Yi L: Role for monoaminergic systems in the antidepressant-like effect of ethanol extracts from Hemerocallis citrina. J Ethnopharmacol 2012, 139(3):780-787.

16. Yi L, Li J, Li H, Zhou Y, Su B, Yang K, Jiang M, Zhang Y: Ethanol extracts from Hemerocallis citrina attenuate the decreases of brain-derived neurotrophic factor. TrkB levels in rat induced by corticosterone administration. J Ethnopharmacol 2012, 144(2):328-334

17. Liu X-L, Luo L, Liu B-B, Li J, Geng D, Liu Q, Yi L-T: Ethanol extracts from Hemerocallis citrina attenuate the upregulation of proinflammatory cytokines and indoleamine 2, 3-dioxygenase in rats. J Ethnopharmacol 2014, 153(2):484-490.

18. Cryan JF, Mombereau C, Vassout A: The tail suspension test as a model for assessing antidepressant activity: review of pharmacological and genetic studies in mice. Neurosci Biobehav R 2005, 29(4):571-625.

19. Colla AR, Machado DG, Bettio LE, Colla G, Magina MD, Brighente I, Rodrigues ALS: Involvement of monoaminergic systems in the antidepressant-like effect of Eugenia brasiliensis Lam. (Myrtaceae) in the tail suspension test in mice. J Ethnopharmaco/ 2012, 143(2):720-731.

20. Machado DG, Cunha MP, Neis VB, Balen GO, Colla A, Bettio LE, Oliveira Á, Pazini FL, Dalmarco JB, Simionatto EL: Antidepressant-like effects of fractions, essential oil, carnosol and betulinic acid isolated from Rosmarinus officinalis L. Food Chem 2013, 136(2):999-1005.

21. Rodrigues ALS, Rocha JBT, Mello CF, Souza DO: Effect of Perinatal Lead Exposure on Rat Behaviour in Open-Field and Two-Wky Avoidance Tasks. Pharmacol Toxicol 1996, 79(3):150-156.

22. Dubois M, Gilles KA, Hamilton JK, Rebers PT, Smith F: Colorimetric method for determination of sugars and related substances. Anal Chem 1956, 28(3):350-356

23. Bradford MM: A rapid and sensitive method for the quantitation of microgram quantities of protein utilizing the principle of protein-dye binding. Anal Biochem 1976, 72(1):248-254.

24. Kim Y, Mosier NS, Hendrickson R, Ezeji T, Blaschek H, Dien B, Cotta M, Dale $B$, Ladisch MR: Composition of corn dry-grind ethanol by-products: DDGS, wet cake, and thin stillage. Bioresour Technol 2008, 99(12):5165-5176.

25. Chang $C$, Yang $M$, Wen $H$, Chern J: Estimation of total flavonoid content in propolis by two complementary colorimetric methods. J Food Drug Anal 2002, 10(3):178-182.

26. Fu M, He Z, Zhao Y, Yang J, Mao L: Antioxidant properties and involved compounds of daylily flowers in relation to maturity. Food Chem 2009, 114(4):1192-1197.

27. Tallarida RJ: Statistical analysis of drug combinations for synergism. Pain 1992, 49(1):93-97.

28. Xia X, Cheng G, Pan Y, Xia Z, Kong L: Behavioral, neurochemical and neuroendocrine effects of the ethanolic extract from Curcuma longa L. in the mouse forced swimming test. J Ethnopharmacol 2007, 110(2):356-363.

29. Lorke D: A new approach to practical acute toxicity testing. Arch Toxicol 1983, 54(4):275-287.

30. Hao C, Lai W, Ho C, Sheen L: Antidepressant-like effect of lemon essential oil is through a modulation in the levels of norepinephrine, dopamine, and serotonin in mice: Use of the tail suspension test. J Funct Foods 2013, 5(1):370-379.
31. Xu Q, Shen $Y$, Wang $H$, Zhang $N$, Xu S, Zhang L: Application of response surface methodology to optimise extraction of flavonoids from fructus sophorae. Food Chem 2013, 138(4):2122-2129.

32. Yang H, Lee Y-C, Han K-S, Singh H, Yoon M, Park J-H, Cho C-W, Cho S: Green and gold kiwifruit peel ethanol extracts potentiate pentobarbital-induced sleep in mice via a GABAergic mechanism. Food Chem 2013, 136(1):160-163.

33. Dimpfel $W$ : Rat electropharmacograms of the flavonoids rutin and quercetin in comparison to those of moclobemide and clinically used reference drugs suggest antidepressive and/or neuroprotective action. Phytomedicine 2009, 16(4):287-294.

34. El-Alfy AT, Ivey K, Robinson K, Ahmed S, Radwan M, Slade D, Khan I, ElSohly $M$, Ross S: Antidepressant-like effect of ${ }^{\Delta 9}$-tetrahydrocannabinol and other cannabinoids isolated from Cannabis sativa L. Pharmacol Biochem Behav 2010, 95(4):434-442

35. Hidalgo M, Sánchez-Moreno C, de Pascual-Teresa S: Flavonoid-flavonoid interaction and its effect on their antioxidant activity. Food Chem 2010, 121(3):691-696.

36. Nutt D: Relationship of neurotransmitters to the symptoms of major depressive disorder. J Clin Psychiatr 2008, 69:4

37. Mao Q, Xian Y, Ip S, Che C: Involvement of serotonergic system in the antidepressant-like effect of piperine. Prog Neuro-Psychopharmacol Biol Psychiatry 2011, 35(4):1144-1147.

doi:10.1186/1472-6882-14-326

Cite this article as: Du et al:: Antidepressant-like effects of the hydroalcoholic extracts of Hemerocallis Citrina and its potential active components. BMC Complementary and Alternative Medicine 2014 14:326.

\section{Submit your next manuscript to BioMed Central and take full advantage of:}

- Convenient online submission

- Thorough peer review

- No space constraints or color figure charges

- Immediate publication on acceptance

- Inclusion in PubMed, CAS, Scopus and Google Scholar

- Research which is freely available for redistribution
C Biomed Central 